\title{
A puzzle on fluctuations of weights in spin glasses
}

\author{
Th. M. Nieuwenhuizen \\ Van der Waals-Zeeman Institute, Universiteit van Amsterdam \\ Valckenierstraat 614/5, 1018 XE Amsterdam, The Netherlands \\ e-mail: nieuwenh@phys.uva.nl \\ (12-4-95; revised 29-9-95; submitted to Journal de Physique I)
}

\begin{abstract}
In certain mean field models for spin glasses there occurs a one step replica symmetry breaking pattern. As an example of general $1 / N$-corrections in such systems, the fluctuations in the internal energy are calculated. For this specific quantity the outcome is known from the specific heat. It is shown that the correct result can be derived by assuming that the both the height and the location of the breakpoint fluctuate. This effect enlarges the commonly considered space of allowed fluctuations of the order parameter in loop calculations for short range spin glasses of this type. The phenomenon does not occur in spin glasses with infinite order replica symmetry breaking.
\end{abstract}

7510.Nr,7540.Cx, 7550.Lk,8710

\section{INTRODUCTION}

The theoretical understanding of the spin glass phase relies on the Parisi replica symmetry breaking scheme. [1] Parisi considered a variational problem with $k$ breakings of replica symmetry. The objects that show up are the values of possible overlaps $q_{i}(0 \leq i \leq k)$ and their cumulative weights $x_{i}(1 \leq i \leq k)$. The free energy was maximized in these variables. In the Sherrington-Kirkpatrick model the physical result arises in the limit $k \rightarrow \infty$. Eliminating the $i$ 's a continuous order parameter function $q(x)$ arises. Loop corrections to this mean field theory have been studied in great detail in a series of papers by de Dominicis, Kondor and Temesvari. [2] In particular, the fluctuation matrix has been inverted in full glory. [3]

Many aspects of this and related replica analyses are ill defined and often ill understood. Nevertheless replica results for the SK-model have been rederived by TAP-analysis and cavity methods. 臿 For the random energy model ("simplest spin glass") the replica results are in full agreement with the direct evaluation of the partition sum. [5]

It is our belief that a replica analysis can produce the physically correct answer in a quick way, but its interpretation should always be understood from a more profound analysis. If the anwer is unsatisfactory, then one should simply abandon it or improve the derivation (the celeber case being Parisi's improvement of the Sherrington-Kirkpatrick saddlepoint.) Such a situation occurs in particular in systems which exhibit one step of replica symmetry breaking (1RSB). Here there occur two transition temperatures, $T_{g}$ for the statics and $T_{c}>T_{g}$ for the dynamics.

Kirkpatrick and Wolynes [6] considered the Potts glass, which exhibits a 1RSB when the number of components exceeds four. In their analysis of the metastable states (TAP states) they observed that there occurs a critical temperature $T_{c}>T_{g}$ where unexpected behavior sets in. On a static level the system condenses for $T_{g}<T<T_{c}$ in a multitude of states with extensive complexity, all of which have non-zero self-overlap but vanishing mutual overlap. The free energy is therefore still equal to the paramagnetic free energy. As a result, this transition is not noticed in a direct replica analysis of the partition sum when one uses Parisi's prescriptions. Nevertheless, it can be described by replica's if one modifies the rule for fixing the breakpoint. The value of the selfoverlap can be obtained by solving the equation $\partial F / \partial q_{1}=0$, with the additional rule that the limit $x_{1} \rightarrow 1$ is taken afterwards. In this temperature interval, namely, it gives the maximal free energy in the physical range $0 \leq x_{1} \leq 1$. Loosely speaking, that limit equates the value of the selfoverlap to the mutual overlaps in a limiting small cluster of states. This procedure also automatically implies that the free energy of this phase is equal to the paramagnetic one. For $T<T_{g}$ the extremum can no longer be located at $x_{1}=1$ and the additional restriction has to be relaxed.

A related mystery of the replica calculus occurs in the dynamical approach to the systems with 1RSB. The prototype is the spherical $p$-spin interaction spin glass, see eq. (1) below. The long-time limit of dynamics has a vanishing fluctuation eigenvalue, and is therefore called "marginal". [7] [8] [9]. The long-time dynamical values for $q_{1}$ and $x_{1}$ can be derived from the replica free energy by assuming marginality within the replica calculation [7] [8] [10]. The mystery remains why a replica calculation can explain the long-time dynamics. After all, the Gibbs weight is thought to be related to exponentially large times where the system has time enough to visit all states. Marginality is thought to be related to algebraically large times. It therefore seems paradoxical that Gibbs weight at marginality does describe the results of long time dynamics. It has been suggested that an essential role is played here by the complexity. It is 
so large that the replica free energy is the lowest of the available states, an old thermodynamic law, but new in glassy systems. 11]

In this note we wish to draw attention to another aspect of the location of the break points of $1 \mathrm{RSB}$ solutions. This time we consider the more standard stationary case, where the Parisi description applies, namely the optimal value of the break points follows by maximizing the free energy. When calculating $1 / N$ contributions to a physical quantity, we find that care should be taken of fluctuations in the location of the plateaus.

In next section we present in detail the calculation of the fluctations of the internal energy in a $p$-spin interaction mean field spin glass model with 1 RSB. Subsequently this analysis is generalized to models with $\infty$ RSB. The paper closes with a discussion.

\section{ANALYSIS OF A MODEL WITH 1RSB}

For a system with $N$ spins in zero field we consider the Hamiltonian

$$
\mathcal{H}=-\sum_{i_{1}<i_{2}<\cdots<i_{p}} J_{i_{1} i_{2} \cdots i_{p}} S_{i_{1}} S_{i_{2}} \cdots S_{i_{p}}
$$

with independent Gaussian random couplings, that have average zero and variance $J^{2} p ! / 2 N^{p-1}$. The spins are spherical and subject to the condition $\sum_{i} S_{i}^{2}=N$. A replica calulation was performed by Crisanti and Sommers (CS). 12] The replicated free energy reads

$$
F_{n}=-\frac{\beta J^{2}}{4} \sum_{\alpha \beta} q_{\alpha \beta}^{p}-\frac{T}{2} \operatorname{tr}_{\alpha} \log q
$$

At zero field the order parameter function has the one-step form $q(x)=q_{1} \theta\left(x-x_{1}\right)$, and the free energy reads

$$
\frac{F}{N}=-\frac{\beta J^{2}}{4}+\frac{\beta J^{2}}{4} \xi_{1} q_{1}^{p}-\frac{T}{2 x_{1}} \log \left(1-\xi_{1} q_{1}\right)+\frac{T \xi_{1}}{2 x_{1}} \log \left(1-q_{1}\right)
$$

where $\xi_{1}=1-x_{1}$. The internal energy reads

$$
U=-\frac{\beta J^{2} N}{2}\left(1-\xi_{1} q_{1}^{p}\right)
$$

Following Crisanti and Sommers in their TAP-approach $[14$, we denote by $z$ the value $0<z<\sqrt{2 / p(p-1)}$ where the complexity

$$
\mathcal{I}=\frac{N}{2}\left(\frac{2-p}{p}-\log \frac{p z^{2}}{2}+\frac{p-1}{2} z^{2}-\frac{2}{p^{2} z^{2}}\right)
$$

is non-extensive, viz. $\mathcal{I} / N=0$. The equations $\partial F / \partial q_{1}=\partial F / \partial x_{1}=0$ then can be cast in the form

$$
\beta(1-q) q_{1}^{p / 2-1}=z ; \quad x_{1}=\frac{\left(2-p z^{2}\right)}{p z^{2}} \frac{1-q_{1}}{q_{1}}
$$

From these results one can calculate the specific heat $C=d U / d T$ using

$$
\frac{d q_{1}}{d T}=\frac{2 \beta q_{1}\left(1-q_{1}\right)}{p-2-p q_{1}}
$$

One obtains

$$
C=\frac{\beta^{2} J^{2} N}{2}\left(1-\xi_{1} q_{1}^{p}-2 q_{1}^{p} \frac{1-\xi_{1}\left(p q_{1}-p+1\right)}{p q_{1}-p+2}\right)
$$

Let us now consider the fluctuations in the internal energy,

$$
\Delta U^{2} \equiv\left\langle\mathcal{H}^{2}\right\rangle-\langle\mathcal{H}\rangle^{2}=\frac{1}{n} \sum_{\alpha, \beta=1}^{n} \int D S \mathcal{H}\left(S_{\alpha}\right) \mathcal{H}\left(S_{\beta}\right) \exp \left(-\beta \sum_{\gamma=1}^{n} \mathcal{H}\left(S_{\gamma}\right)\right)
$$


where the last relation involves replicas, and the limit $n \rightarrow 0$ is implied. The leading terms in $N$ are of order $N^{2} n$, so they donot survive for $n \rightarrow 0$. Indeed, $U$ is expected to have fluctuations of relative order $1 / \sqrt{N}$.

The first $\mathcal{O}(N)$ contribution to $\Delta U^{2}$ comes when all sites in the pre-exponentials overlap pairwise. It yields

$$
\Delta U^{2(1)}=\frac{J^{2} N}{2 n} \sum_{\alpha \beta} q_{\alpha \beta}^{p}=\frac{J^{2} N}{2}\left(1-\xi_{1} q_{1}^{p}\right)
$$

Before calculating the other terms one has to average over the couplings. It implies the shift

$$
J_{i_{1} \cdots i_{p}} \rightarrow J_{i_{1} \cdots i_{p}}+\frac{\beta J^{2} p !}{2 N^{p-1}} \sum_{\gamma} S_{i_{1}}^{\gamma} \cdots S_{i_{p}}^{\gamma}
$$

One gets the contribution

$$
\Delta U^{2(2)}=\frac{\beta^{2} J^{4} N^{2} p ! p !}{4 N^{2 p} n} \sum_{\alpha \beta \gamma \delta} \sum_{i_{1}<i_{2}<\cdots<i_{p}} \sum_{i_{1}^{\prime}<i_{2}^{\prime}<\cdots<i_{p}^{\prime}} \int \frac{d p d q}{2 \pi i} \int D S S_{i_{1}}^{\alpha} S_{i_{1}}^{\gamma} \cdots S_{i_{p}}^{\alpha} S_{i_{p}}^{\gamma} S_{i_{1}^{\prime}}^{\beta} S_{i_{1}^{\prime}}^{\delta} \cdots S_{i_{p}^{\prime}}^{\beta} S_{i_{p}^{\prime}}^{\delta} e^{-\beta \mathcal{H}_{n}}
$$

where the replicated exponential reads

$$
e^{-\beta \mathcal{H}_{n}}=\exp \sum_{\alpha \beta}\left[\frac{N}{4} \beta^{2} J^{2} q_{\alpha \beta}^{p}+\frac{N}{2} p_{\alpha \beta} q_{\alpha \beta}-\frac{1}{2} \sum_{i} p_{\alpha \beta} S_{i}^{\alpha} S_{i}^{\beta}\right]
$$

and $d p d q / 2 \pi i$ is a short hand for the integration measure $\prod_{\alpha<\beta}\left[d p_{\alpha \beta} d q_{\alpha \beta} / 2 \pi i\right] \prod_{\alpha}\left[d p_{\alpha \alpha} / 2 \pi i\right] \times(1+\mathcal{O}(n))$, while further $p$ and $q$ are symmetric. [12] It is indeed seen that the leading terms in $N$ are of order $N^{2} n$, so they donot contribute when $n=0$.

We can now express the prefactors as q's and carry out the spin integrals, to obtain

$$
\Delta U^{2(2)}=\frac{\beta^{2} J^{4} N^{2}}{4 n} \int \frac{d p d q}{2 \pi i} \sum_{\alpha \beta \gamma \delta}\left[q_{\alpha \gamma} q_{\beta \delta}\right]^{p} \exp \left[\sum_{\alpha \beta}\left\{\frac{N}{4} \beta^{2} J^{2} q_{\alpha \beta}^{p}+\frac{N}{2} p_{\alpha \beta} q_{\alpha \beta}\right\}-\frac{N}{2} \operatorname{tr} \log p\right]
$$

Expanding around the saddlepoint one sets $q_{\alpha \beta}=\bar{q}_{\alpha \beta}+\delta q_{\alpha \beta}, p_{\alpha \beta}=\bar{p}_{\alpha \beta}+\delta p_{\alpha \beta}$, with $\delta q_{\alpha \alpha}=0$, where $\bar{q}$ and $\bar{p}=\bar{q}^{-1}$ momentarily denote the saddle point values. One can then integrate out the $\delta p$ 's, so that only the fluctuations in the $q$ 's remain

$$
\Delta U^{2(2)}=\frac{\beta^{2} J^{4} N^{2}}{4 n[\operatorname{det}(\bar{p} \bar{p})]^{1 / 2}} \int d q \sum_{\alpha \beta \gamma \delta} q_{\alpha \gamma}^{p} q_{\beta \delta}^{p} \exp \frac{-N}{2} \sum_{\alpha \beta \gamma \delta} \delta q_{\alpha \beta} M_{\alpha \beta ; \gamma \delta} \delta q_{\gamma \delta}
$$

where $M_{\alpha \beta ; \gamma \delta}=\partial^{2} \beta F_{n} / \partial q_{\alpha \beta} \partial q_{\gamma \delta}$ is the fluctuation matrix. Its matrix elements and eigenvalues are discussed by Crisanti and Sommers [12].

Now one can go to eigenmodes of $M$. [12] The transversal ones can be integrated out, which brings the square root of a another determinant as prefactor. At fixed break point $x_{1}$ only the longitudinal eigenvector $\delta q_{\alpha \beta} \sim q_{\alpha \beta}$ will contribute due to the contraction in the preexponential. However, also $q$ 's with a small shift in the breakpoint will be seen to contribute. [13] We therefore consider fluctuations described by order parameter functions

$$
q_{\alpha \beta} \rightarrow q(x)=(1+\eta) q_{1} \theta\left(x-x_{1}-\delta\right)
$$

where $q_{1}$ and $x_{1}$ are the saddlepoint values, and where $\eta$ gives fluctuations in the height of the plateau and $\delta$ in the location of the breakpoint.

The shape (16) of $q(x)$ leads to $n$-times the free energy (3i) with $q_{1} \rightarrow q_{1}(1+\eta)$ and $x_{1} \rightarrow x_{1}+\delta$. The value at $\delta=\eta=0$ is of order $n$ and has been omitted already from eq. (15). The linear terms vanish due to the saddlepoint condition, and were also omitted already. The quadratic terms therefore yield immediately

$$
\sum_{\alpha \beta \gamma \delta} \delta q_{\alpha \beta} M_{\alpha \beta ; \gamma \delta} \delta q_{\gamma \delta} \rightarrow n\left(\eta^{2} q_{1}^{2} \beta F_{q q}+2 \eta \delta q_{1} \beta F_{q x}+\delta^{2} \beta F_{x x}\right)
$$

We now assume that for $n \rightarrow 0$ the summation over different breakpoints can be replaced by an integral over their location $x_{1}+\delta$ viz. $\int w_{1}\left(x_{1}+\delta\right) d\left(x_{1}+\delta\right)$, where $w_{1}$ is an unknown but smooth weight. Since $\delta$ is of order $1 / \sqrt{N}$, it 
then follows that its weight $w_{1}\left(x_{1}+\delta\right) \approx w\left(x_{1}\right)$ is uniform in $\delta$. The total weight $w$ consists of $w_{1}$ and of square roots of determinants that arise after performing the $\delta p$ integrals and the transversal $\delta q$ modes. Next we assume that $w$ is such that the remaining Gaussian integral over $\delta$ and $\eta$ is normalized to unity. One then has

$$
\Delta U^{2(2)}=\frac{\beta^{2} J^{4} N^{2} n}{4} \frac{\int d \eta d \delta\left[1-\xi_{1} q_{1}^{p}+\delta q_{1}^{p}-p \xi_{1} \eta q_{1}^{p}\right]^{2} \exp \frac{-N n}{2}\left(\eta^{2} q_{1}^{2} \beta F_{q q}+2 \eta \delta q_{1} \beta F_{q x}+\delta^{2} \beta F_{x x}\right)}{\int d \eta d \delta \exp \frac{-N n}{2}\left(\eta^{2} q_{1}^{2} \beta F_{q q}+2 \eta \delta q_{1} \beta F_{q x}+\delta^{2} \beta F_{x x}\right)}
$$

Let us recall that the appearence of the integral in the denominator is due to our assumed value of $w$, now seen to be of order $N n$. As the Gaussian fluctuations bring a factor $1 / N n$, they indeed yield terms that survive in the limit

$n \rightarrow 0$. There are several fluctuation contributions. We should only take into account the cross terms $\left[\delta q_{1}^{p}-p \xi_{1} \eta q_{1}^{p}\right]^{2}$. This yields

$$
\Delta U^{2(2)}=\frac{\beta^{2} J^{4} N q_{1}^{2 p-2}}{4}\left(p^{2} \xi_{1}^{2} R_{q q}-2 p \xi_{1} q_{1} R_{q x}+q_{1}^{2} R_{x x}\right)
$$

where $R$ is the inverse fluctuation matrix, viz. $R_{i j}^{-1}=\beta F_{i j}$, with $i=q, x ; j=q, x$. Inserting the field equations $\partial F / \partial q_{1}=\partial F / \partial x_{1}=0$ one has

$$
\begin{aligned}
\beta F_{q q} & =\frac{-\xi_{1}\left[(p-1) q_{1}-p+2+\xi_{1} q_{1}\left(p-1-p q_{1}\right)\right]}{2\left(1-q_{1}\right)^{2}\left(1-\xi_{1} q_{1}\right)^{2}} \\
\beta F_{q x} & =\frac{\xi_{1} q_{1}^{2}}{2\left(1-q_{1}\right)\left(1-\xi_{1} q_{1}\right)^{2}} \\
\beta F_{x x} & =\frac{-q_{1}^{2}\left(p q_{1}-2 \xi_{1} q_{1}-p+2\right)}{2 p\left(1-q_{1}\right) x_{1}\left(1-\xi_{1} q_{1}\right)^{2}}
\end{aligned}
$$

The inverse matrix reads

$$
\begin{aligned}
R_{i j} & =\frac{-2\left(1-q_{1}\right)\left(1-\xi_{1} q_{1}\right)}{\left(2-p+p q_{1}\right)\left[(p-1) q_{1}-\xi_{1} q_{1}-p+2\right]} S_{i j} \\
S_{q q} & =\frac{1-q_{1}}{\xi_{1}}\left(p q_{1}-2 \xi_{1} q_{1}-p+2\right) \\
S_{q x} & =p x_{1}\left(1-q_{1}\right) \\
S_{x x} & =\frac{p x_{1}}{q_{1}^{2}}\left[(p-1) q_{1}-p+2+\xi_{1} q_{1}\left(p-1-p q_{1}\right)\right]
\end{aligned}
$$

Now one only has to insert these results in (19). One obtains

$$
\Delta U^{2(2)}=-\frac{J^{2} N q_{1}^{p}\left[1-\xi_{1}\left(p q_{1}-p+1\right)\right]}{p q_{1}-p+2}
$$

Note that several unpleasant denominators have canceled, affirming that that our procedure is correct. Putting things together we find

$$
\Delta U^{2}=\Delta U^{2(1)}+\Delta U^{2(2)}=\frac{J^{2} N}{2}\left(1-\xi_{1} q_{1}^{p}-2 q_{1}^{p} \frac{1-\xi_{1}\left(p q_{1}-p+1\right)}{p q_{1}-p+2}\right)
$$

Comparing with eq. (8) one sees that indeed $\Delta U^{2}=T^{2} d U / d T$, as required by a standard thermodynamic relation. If variations in $x_{1}$ had not been taken into account, $\Delta U^{2}$ would have been proportinal to $1 / F_{q q}$, which is certainly incorrect, since the complicated factor in the nummerator of $F_{q q}$ will not be canceled.

\section{INFINITE ORDER REPLICA SYMMETRY BREAKING}

Recently the author proposed a spherical model with infinite order replica symmetry breaking. It is the model given by eq. (17), but summed over $p$. 15] In particular, random pair $(p=2)$ and random quartet interactions $(p=4)$ occur, while random triplets should be absent. Then the model has near the spin glass transition the same critical behavior as the Sherrington-Kirkpatrick model. Its benefit is that it can be solved explicitly in the whole low $T$ phase; 
this arises due to the spherical nature of the spins and the mean field character of the model. For our present purpose this allows an explicit check at any temperature in a model with infinite RSB.

Assuming that the variance of the $p$-spin couplings equals $J_{p}^{2}(p-1) ! / N^{p-1}$, the free energy for a $k$-step RSB pattern can be derived using results of an appendix of CS

$$
\frac{\beta F}{N}=-\frac{1}{4} \beta^{2} f(1)+\frac{1}{4} \beta^{2} \Phi-\frac{1}{2}-\frac{q_{0}}{A_{0}}-\log \left(1-q_{k}\right)-\sum_{i=1}^{k} \frac{1}{x_{i}} \log \frac{A_{i-1}}{A_{i}}
$$

where

$$
f(q)=\sum_{p=2}^{\infty} \frac{J_{p}^{2}}{p} q^{p}
$$

and

$$
\Phi=\sum_{i=0}^{k}\left(x_{i+1}-x_{i}\right) f\left(q_{i}\right) ; \quad A_{i}=1-q_{k}+\sum_{j=i+1}^{k} x_{j}\left(q_{j}-q_{j-1}\right)
$$

As usual we defined $x_{0}=0$ and $x_{k+1}=1$. The variables $\left\{q_{i}, x_{i}\right\}$ will be denoted by a $2 k+1$ component vector $v_{i}$, $(i=0, \cdots, 2 k)$. From $U=-(\beta N / 2)(f(1)-\Phi)$ one obtains by differentiation with respect to $T$ the specific heat

$$
C=\frac{\beta^{2} N}{2}(f(1)-\Phi)+\frac{\beta^{4} N}{4} \sum_{i, j=0}^{2 k} \Phi_{i} R_{i j} \Phi_{j}
$$

where $R_{i j}^{-1}=\partial^{2} \beta F / \partial v_{i} \partial v_{j}$ and $\Phi_{i}=\partial \Phi / \partial v_{i}$.

We again consider the fluctuations in the internal energy. The analog of eq. (10) immediately explains the first two terms of $T^{2} C$. The analog of eq. (19) is

$$
\Delta U^{2(2)}=\frac{\beta^{2} N}{4} \sum_{i, j=0}^{2 k} \Phi_{i} R_{i j} \Phi_{j}
$$

Multiplied by $\beta^{2}$ this indeed equals the last term in eq. (32).

Our approach thus reproduces the correct result for the fluctuations of the internal energy at any fixed $k$ and, in particular, in the physical limit $k \rightarrow \infty$. In this continuum limit the meaning of the breakpoints $x_{i}$ becomes ill-defined. It was conjectured by Parisi [16] that the discussed effect then disappears. In other words, the final result might be due to fluctuations in the plateaus $q_{i}$ only. In order to investigate this question we go to the Parisi free energy functional for the SK model [1]

$$
\beta F=\sum_{i=0}^{k}\left(x_{i+1}-x_{i}\right)\left(\frac{\tau}{2} q_{i}^{2}-\frac{w}{3} q_{i} T_{i}+\frac{y_{1}}{8} q_{i}^{4}\right)
$$

where the paramagnetic background has been subtracted, $\tau=\left(\beta^{2} J^{2}-1\right) / 2$, and

$$
T_{i} \equiv \frac{1}{2}\left(2 x_{i+1}-x_{i}\right) q_{i}^{2}+q_{i} \sum_{j=i+1}^{k}\left(x_{j+1}-x_{j}\right) q_{j}+\frac{1}{2} \sum_{j=0}^{i-1}\left(x_{j+1}-x_{j}\right) q_{j}^{2}
$$

For this model eq. (32) still applies with $f(q)=J^{2} q^{2}$, while the term involving $f(1)=J^{2}$ comes from the paramagnetic background.

The nice thing of the Parisi model is that the solution for $q_{i}, x_{i}$ is known exactly at any $k$. It holds that

$$
q_{i}=\frac{2 i+1}{2 k+1} q_{k} \quad x_{i}=i \frac{6 y_{1} q_{k}}{w(2 k+1)}
$$

where $q_{k}$ satisfies 


$$
y_{1} q_{k}^{2}\left(\frac{3}{2}-\frac{1}{(2 k+1)^{2}}\right)-w q_{k}+\tau=0
$$

This is an immediate, exact generalization of the Parisi solution of the model (34) to the region where $\tau y_{1} / w^{2}$ is not very small.

From Parisi's results it follows that now

$$
U=\frac{\beta J^{2} N}{2} \sum_{i=0}^{k}\left(x_{i+1}-x_{1}\right) q_{i}^{2}=\frac{\beta J^{2} N}{2}\left(q_{k}^{2}-\frac{8 y_{1} k(k+1)}{w(2 k+1)^{2}} q_{k}^{3}\right)
$$

Differentiating with respect to $T$ one finds that $\Delta U^{2(2)}$ should be equal to

$$
\Delta U^{2(2)}=-\frac{\beta^{2} J^{4} N q_{k}}{w} \frac{w-y_{1} q_{k}\left(3-\frac{3}{(2 k+1)^{2}}\right)}{w-y_{1} q_{k}\left(3-\frac{2}{(2 k+1)^{2}}\right)}
$$

Since this expression converges as $1 /(2 k+1)^{2}$, a good approximation is already obtained for $k=1$. The full result for the specific heat converges even as $1 /(2 k+1)^{4}$. [1]

We have also calculated $\Delta U^{2(2)}$ by taking into account only the fluctuations in the $q_{i}$. Numerically we have inverted the matrix $\partial^{2} \beta F / \partial q_{i} \partial q_{j}$ up to large sizes $(k=150)$ and find at $w=y_{1}=1, \tau=0.1$ that

$$
\sum_{i, j=0}^{k} \frac{\partial \Phi}{\partial q_{i}}\left(\frac{\partial^{2} \beta F}{\partial q \partial q}\right)_{i j}^{-1} \frac{\partial \Phi}{\partial q_{j}}=\sum_{i, j=0}^{\infty} \frac{\partial \Phi}{\partial v_{i}}\left(\frac{\partial^{2} \beta F}{\partial v \partial v}\right)_{i j}^{-1} \frac{\partial \Phi}{\partial v_{j}}+\frac{0.446}{(2 k+1)^{3}}=-0.49005929+\frac{0.446}{(2 k+1)^{3}}
$$

where the sum in the middle involves fluctuations in both $q$ and $x$, viz. $\left\{v_{i}\right\}=\left\{q_{0}, q_{1}, q_{2}, \cdots ; x_{1}, x_{2}, \cdots\right\}$.

From this example it is seen that Parisi's conjecture is probably true in general: the effect of fluctuations of the breakpoints disappears for continuous order parameter functions.

\section{CONCLUSION}

We have put the basis for one-loop calculations in a mean field spin glass model with 1RSB. We calculated the energy fluctuations because their strength is known already; they are proportional to the the specific heat. In order to reproduce the correct answer, we had to take into account fluctuations in both the height and the location of the breakpoint of the 1RSB solution. (It should be admitted that the normalization of the fluctuation integral deserves a solid derivation. Our assumed form is the natural one, and leads to the correct results.) 13

It is the new point of this paper that in integrating over all $q_{\alpha \beta}$ one must not only integrate over the fluctuations around the saddlepoint at given parametrization (given breakpoint $x_{1}$ ), but also over all possible parametrizations (locations of the breakpoint). In hinsight, this is not so unnatural. In mean field both the location of the breakpoint and the plateau value are determined by a saddle point equation. Generally saddle points appear when one has to perform sharply peaked integrals. The very notion of a saddle point can only occur due to smallness of fluctuations around it. Saddle points without fluctuations do not exist. Fluctuations must always be considered; only in some cases they are negligible.

The new question which arises is: what happens beyond mean field in finite range systems? Physically, the weight of the 1RSB solution is the probability for occurrence of non-zero overlap. At before hand, there is no reason why it should be constant. One would thus expect that it fluctuates. This is exactly what we showed in an explicit mean field calculation. Beyond mean field one expects that it fluctuates in space. In the renormalization group approach of Cwilich and Kirkpatrick [17] such fluctuations are not taken into account.

An important question is whether they are compatible with ultrametricity. The answer is affirmative. Indeed, given Parisi's division of the replica number $n=\left(n / x_{1}\right)\left(x_{1} / x_{2}\right) \cdots\left(x_{k} / 1\right)$, one can impose at each lattice sites the allowed "ultrametric states" $x_{1}, \cdots, x_{k}$. In mean field only one "state", say $\bar{x}$, will be occupied; this happens uniformly in space. For describing fluctuations of the breakpoint at site $r$, the state with breakpoint $x(r)=\bar{x}+\delta x(r)$ will be occupied at the cost of the occupation of the state with $\bar{x}$. This will modify the longitudinal sector of the fluctuations. As this sector is massive at the transition and below, it is expected to bring only quantitative effects, and not to change the renormalization flow. 117].

In models with infinite order replica symmetry breaking the order parameter function has in zero field a continuous part up to $x=x_{1}$ and is constant beyond. Here the effect of fluctuations in the location of the breakpoints was seen 
to disappear. For short range systems this implies that the breakpoints donot fluctuate in space. The fluctuations of the probability of the self-overlap, i.e. $1-x_{1}$, are probably already taken into account by the fluctuations in the height of the plateau.

\section{ACKNOWLEDGMENTS}

The author benefited from participation in the conference "Low temperature dynamics and phase-space structure of complex systems", held at Nordita, Kopenhagen, March 1995. He also acknowledges discussion with J.M. Luck and hospitality at the CEA Saclay, where part of the work was done. He thanks Giorgio Parisi for urging him to publish this material. This work was made possible by the Royal Dutch Academy of Arts and Sciences (KNAW).

[1] G. Parisi, J. Phys. A 13 (1980) L115; ibid. 1101

[2] C. de Dominicis and I. Kondor, Phys. Rev. B27 (1983) 606; C. de Dominicis, I. Kondor and T. Temesvari, Int. Journ. Mod. Phys. B 7 (1993) 986;

[3] C. de Dominicis, I. Kondor and T. Temesvari, J. Phys. I France 4 (1994) 1287

[4] M. Mézard, G. Parisi, and M.A. Virasoro, Spin Glass Theory and Beyond (World Scientific, Singapore, 1987)

[5] D.J. Gross and M. Mézard, Nuclear Phys. B240 [FS12] (1984) 431

[6] T.R. Kirkpatrick and P.G. Wolynes, Phys. Rev. B 36 (1987) 8552

[7] T.R. Kirkpatrick and D. Thirumalai, Phys. Rev. Lett. 58 (1987) 2091; Phys. Rev. B 36 (1987) 5388

[8] A. Crisanti, H. Horner, and H.J. Sommers, Z. Phys. B 92 (1993) 257

[9] L. F. Cugliandolo and J. Kurchan, Phys. Rev. Lett. 71 (1993) 173

[10] Th.M. Nieuwenhuizen, Phys. Rev. Lett. 74 (1995) 3463

[11] Th.M. Nieuwenhuizen, Complexity as the driving force for dynamical glassy transitions, preprint (March, 1995); condmat/9504059

[12] A. Crisanti and H.J. Sommers, Z. Physik B 87 (1992) 341

[13] As a certain linear combination of 'replicon' or 'ergodon' fluctuations also represents a shift in the breakpoint, this may look like overcounting the fluctuations. However, eq. (15) only describes the small, Gaussian fluctuations. After completion of the manuscript, it was realized that eq. (14) should have fluctuations $\delta p \sim \delta q \sim \mathcal{O}\left(N^{0}\right)$, that are effectively taken into account by our shift in $x_{1}$. We thank M. Ferrero and an unknown referee for discussion on this item.

[14] A. Crisanti and H.J. Sommers, J. Phys. I (France) 5 (1995) 805

[15] Th.M. Nieuwenhuizen, Phys. Rev. Lett. 74 (1995) 4289

[16] G. Parisi, private communication (March, 1995)

[17] G. Cwilich and T.R. Kirkpatrick, J. Phys. A 22 (1989) 4971; G. Cwilich, J. Phys. A 23 (1990) 5029 